\title{
Neuroectodermal (CHIME) syndrome: an additional case with long term follow up of all reported cases
}

\author{
Vandana Shashi, Janice Zunich, Thaddeus E Kelly, Julie S Fryburg
}

\begin{abstract}
A new neuroectodermal syndrome (designated CHIME syndrome) was described in 1983 with a total of four patients reported, it is presumed to be an autosomal recessive disorder because of recurrence in sibs. The main features include ocular colobomas, congenital heart disease, early onset migratory ichthyosiform dermatosis, mental retardation, conductive hearing loss, seizures, and typical facial features. We report a fifth child with the condition, confirming the unique nature of the condition. Long term follow up information on this patient, as well as the previously described cases, provides information regarding the outcome for these patients, which includes general good health, severe mental retardation, seizures that worsen after puberty, conductive hearing loss, and chronic migratory ichthyosiform skin rash without scarring.
\end{abstract}

(f Med Genet 1995;32:465-469)

A new neuroectodermal syndrome (CHIME syndrome) was reported by Zunich and Kaye ${ }^{1}$ in 1983 with a total of four patients reported. ${ }^{2-4}$ Ichthyosis and neurological abnormalities including seizures and mental retardation have been described in several genetic syndromes. These include Rud syndrome, ${ }^{5}$ Refsum disease, ${ }^{6}$ Sjögren-Larsson syndrome, ${ }^{7}$ Netherton syndrome, ${ }^{8} k$ eratitis, $i$ chthyosis and deafness (KID) syndrome, ${ }^{910}$ and $i$ chthyosis, brittle hair, impaired intelligence, decreased fertility, and short stature (IBIDS) syndrome. ${ }^{11}$ Other abnormalities such as hearing loss, eye abnormalities, and hypogonadism have been reported in some of these disorders. Although there is some overlap in the clinical features, certain clinical characteristics help to distinguish one disorder from another.

A new neuroectodermal syndrome distinct from previously known entities was described in a patient by Zunich and Kaye ${ }^{1}$ in 1983. Subsequently, two other cases and a probable fourth were reported by the same authors. ${ }^{2-4}$ The main features consist of ocular colobomas, migratory ichthyosiform rash, heart defect, mental retardation, seizures, conductive hearing loss, and typical facial features including ocular hypertelorism, epicanthic folds, flat nasal root, full lips, and abnormal dentition. The four children were from infancy to age 6 years at the time of reporting. The authors suggested the name "CHIME" syndrome, derived from ocular colobomas heart defect, $i$ chthyosis, mental retardation, and abnormal ears. We report an additional child with this syndrome, as well as follow up information on the present and the four previously reported patients. This provides information regarding the prognosis for the first two decades of life for people with this condition.

\section{Case reports}

CURRENT PATIENT (PATIENT 5, TABLE)

A term female infant was born by spontaneous vaginal delivery after a normal pregnancy to a 20 year old primigravida. The birth weight was $3500 \mathrm{~g}$ (75th centile) and the length was $52.5 \mathrm{~cm}$ (95th centile). Dryness of the skin and an erythematous papular rash on the neck and trunk were present at birth. Ocular hypertelorism and a webbed neck were noted. By the age of 1 month, the skin rash was migratory in nature, involving the trunk and the limbs, and persistent in spite of application of moisturisers and steroid creams. At this time she developed respiratory distress. Echocardiogram and cardiac catheterisation showed a large ventricular septal defect with severe pulmonary hypertension, which was surgically corrected at $2 \frac{1}{2}$ years. Ultrasound studies of the abdomen showed left hydronephrosis owing to ureteropelvic junction (UPJ) obstruction, which was repaired by pyeloplasty at 15 months. The left hip was found to be subluxated and responded to splinting. Eye examination during infancy showed hyperopia and bilateral incomplete choroidal colobomas. On audiological testing she had mild conductive hearing loss. At 8 months, because of persistent pulmonary infiltrates following several respiratory infections, she underwent a lung biopsy that was reported to be normal.

At the age of 6 months she developed myoclonic seizures that were poorly responsive to anticonvulsant medication. An EEG performed at 2 years of age showed frequent and independent multifocal bilateral epileptiform discharges in the right and left parietal and posterotemporal areas. All developmental milestones were delayed. She walked at 18 months, spoke her first words at 2 years, and was toilet trained at 5 years. There was difficulty with feeding in the first few years with frequent choking on solid foods; this resolved completely by the age of 6 years.

Blood lymphocyte karyotype on two occasions, plasma amino acids, very long chain 
Clinical features of present patient (5) compared to the previously reported cases of neuroectodermal syndrome

\begin{tabular}{|c|c|c|c|c|}
\hline Features & Patient $1^{*}$ & Patient 2 & Patient $3^{*}$ & Patient 5t \\
\hline Age when reported & $4 y$ & $6 \frac{1}{2} y$ & $6 \mathrm{~m}$ & $15 y$ \\
\hline Current age & $16 y$ & $18 \mathrm{y}$ & $7 y$ & $15 y$ \\
\hline $\begin{array}{l}\text { Sex } \\
\text { Ichthyosis/rash } \ddagger\end{array}$ & $M$ & $\mathrm{~F}$ & M & F \\
\hline \multicolumn{5}{|l|}{ Hair } \\
\hline Sparse, blond, fine & + & + & + & + \\
\hline Eye colobomas & + & + & + & + \\
\hline \multicolumn{5}{|l|}{ Facial } \\
\hline $\begin{array}{l}\text { Ocular hypertelorism } \\
\text { Brachycephaly }\end{array}$ & + & + & + & + \\
\hline $\begin{array}{l}\text { Brachycephaly } \\
\text { Epicanthic folds }\end{array}$ & + & + & + & + \\
\hline $\begin{array}{l}\text { Epicanthic folds } \\
\text { Flat, broad nasal root }\end{array}$ & + & + & + & + \\
\hline $\begin{array}{l}\text { Flat, broad nasal root } \\
\text { Oral }\end{array}$ & + & + & + & + \\
\hline \multicolumn{5}{|l|}{ Oral } \\
\hline Full lips & + & + & + & + \\
\hline $\begin{array}{l}\text { Cleft palate } \\
\text { Teeth }\end{array}$ & + & - & + & - \\
\hline Widely spaced & \multicolumn{2}{|c|}{ Teeth } & + & + \\
\hline Bifid/extra incisors & + & + & + & $\stackrel{+}{-}$ \\
\hline \multicolumn{5}{|l|}{ Ears } \\
\hline Overfolded helices & + & + & + & + \\
\hline Conductive deafness & + & + & + & + \\
\hline Congenital heart disease & - & + & + & + \\
\hline \multicolumn{5}{|l|}{ Extremities } \\
\hline Broad second toe & + & + & + & + \\
\hline Thickened palms and soles & + & + & + & + \\
\hline \multicolumn{5}{|l|}{ CNS } \\
\hline Mental retardation & + & + & + & + \\
\hline Seizures & + & + & + & + \\
\hline Abnormal gait & + & + & + & + \\
\hline Cerebral atrophy & + & + & - & - \\
\hline Renal abnormalities & - & - & - & + \\
\hline
\end{tabular}

* Patients 1 and 3 are brothers. Patient 4 is not included in table because of insufficient details. + Most recent patient reported.

$¥$ Age when ichthyosis/rash became manifest.

CNS = central nervous system.

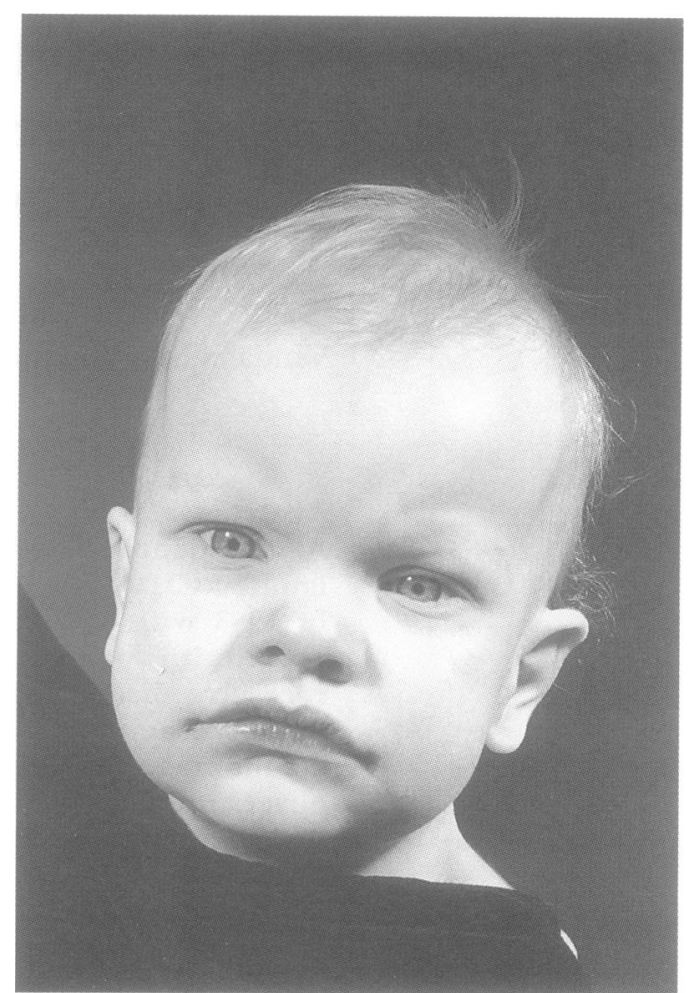

Figure 1 Patient 5 aged 2 years. Sparse and fine scalp hair, hypertelorism, epicanthic folds, flat, broad nasal bridge, wide mouth, and full lips are evident.

fatty acids, and urine organic acids were normal. Molecular cytogenetic analysis by FISH and DNA analysis with probes in the Angelman/Prader-Willi region excluded both a deletion and uniparental disomy of the 15q1112 region. A CT scan of the head at 4 years of age showed no abnormalities. Radiographs of the cervical and thoracolumbar spine were normal.

Her growth began to decelerate in the second year of life, with height and weight dropping to the 10th centile. The head circumference dropped from the 50th centile in the first year to the 5 th by 2 years to below the 5 th centile after 2 years. Physical examination at 22 months (fig 1) showed a flattened occiput, sparse, light, and fine scalp hair, a prominent forehead, ocular hypertelorism, a broad and flat nasal bridge, a wide mouth, and delayed development.

Her parents are healthy and non-consanguineous. She has six sibs, with no history of mental retardation, seizures, skin disease, congenital heart disease, hearing loss, or dysmorphic features.

At the time of the most recent examination, aged $14 \frac{1}{2}$ years, she weighed $39 \mathrm{~kg}$ ( 5 th centile), was $152.5 \mathrm{~cm}$ tall (10th centile), with a head circumference of $45 \mathrm{~cm}$ ( $<5$ th centile). Occipital flattening, prominent forehead, and lightly coloured, sparse, and fine scalp hair, among other dysmorphic features, were noted (fig 2, table). Squamous debris was seen in the ear canals. The neck was webbed. The skin was extremely dry, and areas of excoriation and papular rash were apparent on the trunk, with thickening of the skin of the palms and feet. The hands and feet appeared large with prominent interphalangeal joints and broad second toes. Bilateral fifth finger camptodactyly and clinodactyly were noted. Dermatoglyphics and dermal ridges were normal. Secondary sexual characteristics were Tanner stage II. On neurological examination she had normal tone, strength, and deep tendon reflexes. The gait was wide based and unsteady. Formal psychological testing at the age of 14 showed that cognitive skills were between a 3 and $4 \frac{1}{2}$ year level, with speech most severely affected at a 22 month level.

Currently, the patient is $15 \frac{1}{2}$ years old. Her parents report that she communicates mostly using sign language, with her vocabulary limited to 10 to 15 words. She continues to have exfoliation of skin in the external auditory canal. Recurrent middle ear infections necessitated placement of ventilatory tubes on three occasions. She wears glasses for hyperopia; the ocular findings have not changed over time. Dryness of the skin has persisted, with the periodic appearance of a maculopapular rash mainly on the face, trunk, and lower extremities. Episodes of violent behaviour including kicking, screaming, and hitting family members appear to correlate with the exacerbation of the skin rash, and also noted to be worse since puberty. Healing of affected skin areas occurs without scarring. Corticosteroid creams have been of little benefit. Intensive moisturising treatments are of some benefit. Since the age of 2 , the seizures have been of grand mal type and the frequency of seizures years. Currently on valproic acid, she continues to have eight to ten seizures per month and is under the care of a neurologist. A recent EEG showed findings similar to the previous one. There have been no complications following heart surgery, with almost complete resolution of pulmonary hypertension. has increased since menarche, attained at 15 


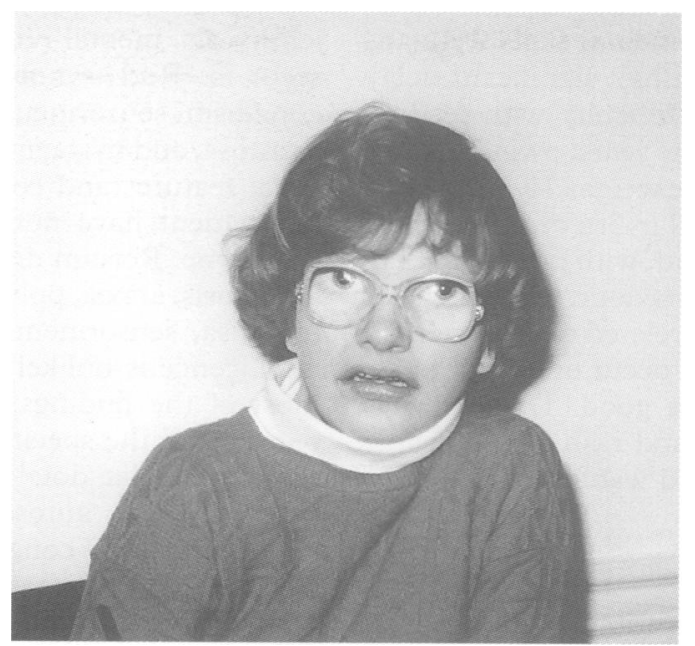

Figure 2 Patient 5 aged 15 years. Note the hypertelorism, broad nasal bridge, full lips and widely spaced teeth.

\section{PREVIOUSLY REPORTED CASES ${ }^{1-4}$ \\ Patient 1 (table)}

The first patient described was a male, born at term, with weight and length above the 90th centile. Brachycephaly and cleft palate were noted. At 1 week of age, he developed a pruritic migratory ichthyosiform rash. Seizures began at 9 months of age. At $3 \frac{1}{2}$ years of age, he was found to be developmentally delayed at a 12 month level, with persistence of the migratory ichthyosiform rash. His hair was blond, sparse, and fine. Other features included retinal colobomas, epicanthic folds, broad and flat nasal root, overfolded helices of the ears, full lips, small irregularly spaced teeth with a bifid incisor, broad second toes, excess digital arch pattern, and wide based gait. Conductive deafness was found resulting from exfoliation of skin in the ear canal. Blood lymphocyte karyotype was normal. A CT scan of the head showed mild cerebral atrophy. A diagnosis of a previously unknown "neuroectodermal" syndrome was made. ${ }^{1}$

His physical growth continued to be good, despite difficulty in feeding owing to poor coordination of swallowing in the first decade of life. Delays in his development became more pronounced with age. General health has been good, except for frequent sinus infections. Currently, at the age of 16 years, he is $1.72 \mathrm{~m}$ tall (25th centile) and weighs $61 \mathrm{~kg}$ (25th centile). $\mathrm{He}$ is profoundly mentally retarded with no speech and the only means of communication is by pictures. Seizures have worsened over the years in spite of rigorous anticonvulsant therapy. The skin rash has continued to remit and relapse over the years. The dryness has also persisted. All topical treatment regimens have been of limited value. There is thickening of the palms and soles. He too has episodes of violent behaviour correlated with exacerbation of the skin rash, as seen in patient 5. Also, the behavioural outbursts have been worse since puberty. The conductive deafness has persisted. Sexual development has been normal.

His parents are non-consanguineous. An older sister is healthy. Subsequently, his younger brother (patient 3) was born with the same condition.

\section{Patient 2 (table)}

This $6 \frac{1}{2}$ year old female was born after a normal pregnancy, at term, with height and weight about the 50th centile. Tetralogy of Fallot and left sided club foot were obvious in the neonatal period. At the age of 1 month, a migratory ichthyosiform rash developed. Seizures began at 8 months of age and were found to be worse with fever. Moderate conductive hearing loss was felt to be because of exfoliation of skin in the external ear canal. Bilateral retinal colobomas were detected on eye examination. Ocular hypertelorism, epicanthic folds, broad and flat nasal root, brachycephaly, overfolded helices of the ear, full lips, and small irregularly spaced teeth with an extra incisor were present. Scalp hair was blond, fine, and sparse. Broad second toes, brachydactyly, and an excess of arches on the fingers were noted. Palms and soles were thickened. The gait was wide based. There was an erythematous, scaly eruption, but the uninvolved skin appeared normal. Her developmental skills at the age of 6 were at a 18 to 24 month level. CT scan of the head showed mild cerebral atrophy.

The heart defect was surgically corrected. Chromosomal analysis and tests of immune function were normal. Physical growth continued to be good with height and weight around the 90th centile. Currently, at the age of 18 years, she is $1.72 \mathrm{~m}$ tall (90th centile) and weighs $57 \mathrm{~kg}$ (50th centile). Mental retardation is profound with no speech, and the only means of communicating is with pictures. Outbursts of violent behaviour occur periodically and seem to have worsened since puberty which occurred at a normal time. Seizures have continued to worsen in frequency despite anticonvulsant therapy. The deafness has persisted. General health is good except for frequent sinus infections. The skin rash continues to recur in a migratory fashion. The gait is still wide based. There have been no problems related to the repaired heart defect.

Her parents are unrelated and healthy; an older sister is normal.

\section{Patient 3 (table)}

A 6 month old male, with height and weight above the 90th centile, was born to the parents of patient 1, nine years later. Transposition of the great vessels was repaired by balloon septostomy and Jatene procedure at 3 weeks of age. Seizures began in the postoperative period. In addition, a flat and broad nasal root, small ears with overfolded helices, submucous cleft palate, and dryness of the skin were noted. Conductive hearing loss and retinal colobomas were detected. CT scan of the head was normal, as was blood lymphocyte karyotype.

On examination over the next few years, several dysmorphic features not included in the original report were noted, including brachycephaly, hypertelorism, epicanthic folds, broad and flat nasal root, full lips, small and irregularly spaced teeth with bifid upper and lower incisors, and broad second toes. The palms and soles were thickened and the gait was wide based. Although at the time of the 
initial report his developmental skills were appropriate for age ( 4 months), significant delay later became obvious. Difficulty with feeding was present in the first few years, owing to poor swallowing. At 7 years, he weighs $19.5 \mathrm{~kg}(<5$ th centile) and is $1.09 \mathrm{~m}$ tall ( $<5$ th centile). Mental retardation is profound, with no speech. $\mathrm{He}$ exhibits self-abusive behaviour. Outbursts of violent behaviour are correlated with worsening of his skin rash. Seizures occur only with fever. General health has been good. Deafness has persisted. Skin dryness and rash are a chronic problem with waxing and waning.

\section{Patient 4}

In a personal communication to the original authors, ${ }^{4}$ a male infant was described to have extensive migratory ichthyosiform rash, retinal colobomas, hearing loss, peripheral pulmonary stenosis, and severe developmental delay. No further information is available on the patient. $\mathrm{He}$ died at 1 year from pneumonia.

\section{Discussion}

A new "neuroectodermal syndrome" was reported in three patients by one of us, ${ }^{1-4}$ with a fourth case in a personal communication. ${ }^{4}$ The occurrence in two sibs and in both sexes suggested autosomal recessive inheritance. We present the fifth documented case of this entity providing evidence confirming the unique nature of this condition and information regarding the outcome for the first two decades of life.

The features in our patient of early onset migratory ichthyosiform dermatosis, mental retardation, seizures, ocular colobomas, conductive hearing loss, wide based gait, and craniofacial features consisting of brachycephaly, sparse, light, and fine scalp hair, hypertelorism, epicanthic folds, broad, flat nasal bridge, overfolded ear helices, full lips, and wide mouth with irregularly spaced teeth are similar to the previous three cases reported by Zunich $e t a l,{ }^{1-4}$ as are the presence of broad second toes and thickened palms and soles. In addition, congenital heart disease, found in our patient and in three of the four previous cases, is probably a component of this syndrome. While our patient has most features consistent with the previous cases, there are a few differences (table). She has choroidal colobomas while the previous cases had retinal colobomas. UPJ obstruction, seen in our case, has not been previously reported; this may be incidental and unrelated to the condition. Our patient also does not have cleft palate reported in the two familial cases, ${ }^{4}$ though the cleft palate in the two brothers may be the result of familial factors unrelated to neuroectodermal syndrome. Cerebral atrophy, detected in two of the previous cases, was absent in patient 5 . These minor differences may be attributable to variability within the neuroectodermal syndrome.

We considered several disorders with ichthyosis and neurological abnormalities in the differential diagnosis of our patient and found features that clearly distinguish her condition from these. Although our patient has congenital ichthyosis, mental retardation, and seizures as seen in Rud syndrome, ${ }^{5}$ she lacks hypogonadism, sensorineural deafness, retinitis pigmentosa, and nystagmus. Moreover, the typical facial features and congenital heart disease in our patient have not been described in Rud syndrome. Refsum disease, ${ }^{612}$ characterised by ichthyosis, ataxia, polyneuropathy, retinitis pigmentosa, sensorineural deafness, and normal intelligence is unlikely, since our patient lacks most of the findings. The absence of spastic paresis and the specific ocular changes of glistening macular dots ${ }^{13}$ makes Sjögren-Larsson syndrome, an autosomal recessive disorder characterised by congenital ichthyosis, mental retardation, and spastic paresis, ${ }^{7}$ unlikely. In Netherton syndrome, congenital ichthyosiform dermatosis, sparse hair, and mental retardation are seen ${ }^{814}$; however, the ocular changes, congenital heart disease, and typical facies seen in our patient have not been described. The sensorineural deafness and keratitis seen in KID syndrome ${ }^{10}$ are notably absent in our case. IBIDS syndrome ${ }^{1115}$ is unlikely, owing to the absence of hypogonadism as well as cataracts which have been frequently described in this disorder. The findings of microcephaly, occipital flattening, light coloured hair, wide mouth with widely spaced teeth, mental retardation, seizures, and unsteady gait are all compatible with Angelman syndrome. However, her dermatological findings, sparse hair, congenital heart disease, ocular colobomas, hearing loss, and language acquisition are not features usually seen in Angelman syndrome. In addition, chromosome and molecular analyses were normal.

Patient 4, described in a personal communication, ${ }^{4}$ died in infancy from pneumonia. Clinical follow up to the age of 15 years in patient 5 , as well as up to ages 16,18 , and 7 years, respectively, in the other three cases has provided some information regarding the long term outlook for people with this rare condition. Significant mental retardation, seizures, and chronic migratory ichthyosiform dermatosis are the major problems in the first and second decades of life. General health is good, after correction of the heart defect, though these patients experience frequent respiratory infections in the first few years of life. Puberty occurred at a normal age in patients 1,2 , and 5 ; we believe that hypogonadism is not a feature of this syndrome. Severe to profound mental retardation, along with outbursts of violent behaviour, have resulted in the need for constant supervision. These episodes appear to be worse after puberty and are correlated with exacerbation of the skin rash. Inability to deal with the pruritis associated with the skin rash may be contributing to the behavioural outbursts. Of the four, only patient 5 has acquired the ability to speak; this lack of verbal skills is likely to be the result of the severe mental impairment, although the long standing conductive deafness may be a contributing factor. Seizures have worsened in patients 1,2 , and 5 after puberty, and are poorly controlled in spite of aggressive anticonvulsant therapy. The reason for this worsening with puberty is un- 
clear, although hormonal changes may be responsible. Patient 3, who at the age of 7 , has seizures only with fever, may experience worsening after puberty. The skin rash is recurrent, with exacerbations and remissions. Topical treatments of various kinds have only a palliative effect.

The underlying defect in some of the syndromes with ichthyosis and neurological abnormalities is known: Sjögren-Larsson syndrome is caused by a defect in the metabolism of essential fatty $\operatorname{acid}^{16}$; in Refsum's disease, owing to deficiency of alpha-hydroxylase, there is accumulation of phytanic acid, a branched chain fatty acid. ${ }^{17}$ We thus speculate that there may be an as yet unknown defect in fatty acid metabolism in the patients described in this report. Although occurrence in sibs prompted the initial authors to propose autosomal recessive inheritance, alternative mechanisms including gonadal mosaicism and uniparental disomy cannot be excluded.

The acronym "CHIME" to describe this syndrome was suggested by the initial authors and cited as such. ${ }^{18}$ This was drawn from the main features namely, ocular colobomas, heart defect, $i$ chthyosiform dermatosis, mental retardation, and ear anomalies. We suggest that this condition be designated "CHIME syndrome".

In conclusion, this is the fifth case of the "CHIME" syndrome first described by Zunich and Kaye. ${ }^{1}$ The presence of ocular colobomas, congenital heart disease, early onset ichthyosiform dermatosis, mental retardation, seizures, conductive hearing loss, and typical facial features in our patient, as well as the four previously reported cases, strengthens the unique nature of this syndrome. This disorder should be considered in the differential diagnosis of a child with mental retardation and ichthyosis. The outlook for the first and second decades includes general good health if the heart defect is corrected, severe to profound mental retardation, seizure disorder that worsens after puberty, chronic ichthyosiform dermatosis, and hearing loss. More case reports are needed to delineate the natural history of this disorder further.

1 Zunich J, Kaye CI. New syndrome of congenital ichthyosis with neurologic abnormalities. Am f Med Genet 1983;15: $331-3$

2 Zunich J, Kaye CI. Additional case report of new neuroectodermal syndrome. Am f Med Genet 1984;17:707-10.

3 Zunich J, Esterly NB, Holbrook KA, Kaye CI. Congenital migratory ichthyosiform dermatosis with neurologic and ophthalmologic abnormalities. Arch Dermatol 1985;121: 1149-56.

4 Zunich J, Esterly NB, Kaye CI. Autosomal recessive transmission of neuroectodermal syndrome. Arch Dermatol 1988;124:1188-9.

5. Marxmiller J, Trenkle I, Ashwal S. Rud syndrome revisited: ichthyosis, mental retardation, epilepsy and hypogonadism. Dev Med Child Neurol 1985;27:335-43.

6 Steinberg G. Phytanic acid storage disease (Refsum's syndrome). In: Stanbury JB, Wyngaarden JB, Frederickson drome). In: Stanbury JB, Wyngaarden JB, Frederickson DS, eds. The metabolic basis of in

7 Jagell S, Gustavson KH, Holmgren G. Sjögren-Larsson syndrome in Sweden: a clinical, genetic and epidemiological study. Clin Genet 1981;19:233-56.

8 Altman J, Strand J. Netherton's syndrome and ichthyosis linearis circumflexa. Arch Dermatol 1969;100:556-8

9 Senter TP, Jones KL, Sakati N, Nyhan WL. Atypical ichthyosiform erythroderma and congenital neurosensory deafness-a distinct syndrome. F Pediatr 1978;92:69-72.

10 Skinner BA, Greist MC, Norins AL. The keratitis, ichthyosis and deafness (KID) syndrome. Arch Dermatol 1981;117: $285-9$.

11 Rebora A, Crovato F. Trichothiodystrophy, xeroderma pigmentosum and PIBI(D)S syndrome. Hum Genet 1988;78: 106-8.

12 Refsum S. Heredopathia atactica polyneuritiformis, Refsum's disease: a biochemically well-defined disease with a specific dietary treatment. Arch Neurol 1981;38:605-6. Jagell S, Polland W, Sandgren O. Specific changes in the fundus typical for the Sjögren-Larsson syndrome: an oph-
thalmological study of 35 patients. Acta Ophthalmol 1980; 59:321-30.

14 Greene SL, Muller SA. Netherton's syndrome: report of a case and review of the literature. $\mathcal{F}$ Am Acad Dermatol 1985;13:329-37

15 Son Blomquist HK, Back O, Fagerlund M, Holmgren G, Stecksen-Blicks C. Tay or IBIDS syndrome: a case with growth and mental retardation, congenital ichthyosis and brittle hair. Acta Paediatr Scand 1991;80:1241-5.

16 Hernell O, Holmgren G, Jagell SF, Johnson SB, Holman RT. Suspected faulty essential fatty acid metabolism in Sjögren-Larsson syndrome. Pediatr Res 1982;16:45-9.

17 Herndon JH, Steinberg D, Uhlendorf BW, Fales HM. Refsum's disease: characterization of the enzyme defect in cell culture. $f$ Clin Invest 1969;48:1017-32.

18 Ladda RL, Zunich J. Ichthyosis-colobomas-heart defectdeafness-mental retardation. In: Buyse $M L$, ed. Birth defects encyclopedia. Cambridge: Blackwell, 1990:3214, defects
945. 